\section{Of mice and men}

\author{
Reviewed by Michael Stoskopf, DVM, PhD, D.ACZM
}

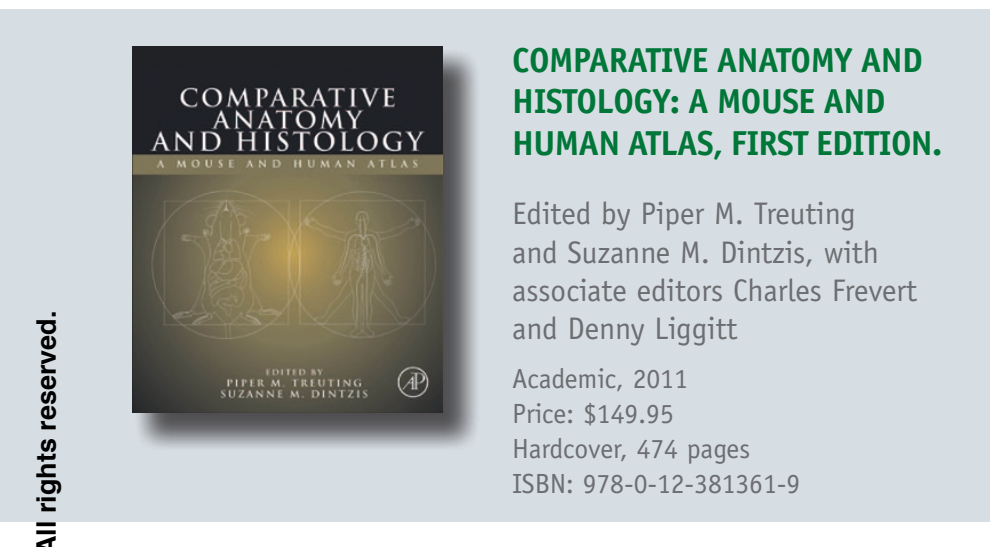

The editors and authors of this book have created a useful reference that can serve as a text for teaching the important comparative aspects of human and mouse histology and anatomy to medical and veterinary pathologists, as well as researchers using mouse models. Twenty-three chapters, organized by system and authored by a well-qualified stable of experts, provide a very comprehensive text, strengthened by large numbers of gross and microscopic images throughout the work. The juxtaposition of images and text for each species throughout the book provides a unique perspective that sets a new standard for comparative atlases. The extensive use of very well-rendered medical illustrations to supplement the highquality microscopic and dissection photography will make this book a classic resource for pathologists, researchers and laboratory animal specialists throughout their careers.

The scope and completeness of this work makes an exhaustive review of every chapter or feature beyond the scope of any one reviewer, but the editors have done a yeoman's job in striving for both completeness and consistent presentation. Perhaps as important, there is significant synthesis of information throughout the book. An excellent example is Table 1 in the introduction, which compares general features of the taxonomy, size and organ organization of two species in side-by-side columns. The introductory paragraph also provides an excellent discussion of the importance of comparative pathology when translating experimental findings in mice to human disease. The second chapter on Phenotyping, by Cory Brayton and Piper Treuting, provides a well-organized discussion of why anatomic research remains relevant and a concise tutorial pointing out key elements to consider when designing studies using mouse models of human conditions. This is followed by a well-written chapter on necropsy and histology techniques, provided by Sue Knoblaugh, Lulie Randolph-Habecker and Steve Wrath, that will find broad use in introductory training for new investigators and pathologists.

From there, an excellent collection of system- or organbased chapters follows, each with subsections on gross anatomy and histology. Covered topics include the skeletal, respiratory, cardiovascular, gastrointestinal, endocrine, reproductive and nervous systems, as well as teeth, liver, hematopoietic and lymphoid tissues, skin and sensory organs. These chapters are thoroughly illustrated, contain many helpful tables to summarize the most relevant information and use helpful "Need to Know" call outs that serve to emphasize key points throughout the book. Each chapter also ends with a "Further Reading" section, which provides useful references for topics covered in the chapter.

The availability of the book online in the Expertconsult.com resource, managed by Elsevier, or as a Kindle text makes using this important resource even more convenient for scientists with different preferences in the delivery of their reference sources. This is a book that many scientists from a wide range of disciplines will want to have on their shelves or computers long into the future. 